\title{
Addressing Inpatient Glycaemic Control with an Inpatient Glucometry Alert System
}

\author{
J. N. Seheult, ${ }^{1}$ A. Pazderska, ${ }^{2}$ P. Gaffney, ${ }^{1}$ J. Fogarty, ${ }^{1}$ M. Sherlock, ${ }^{2}$ \\ J. Gibney, ${ }^{2}$ and G. Boran ${ }^{1}$ \\ ${ }^{1}$ Clinical Chemistry Department, Adelaide and Meath Hospital, Tallaght, Dublin 24, Ireland \\ ${ }^{2}$ Department of Medicine, Endocrinology Division, Adelaide and Meath Hospital, Tallaght, Dublin 24, Ireland
}

Correspondence should be addressed to J. N. Seheult; seheultj@tcd.ie

Received 6 November 2014; Revised 27 May 2015; Accepted 8 June 2015

Academic Editor: Andre P. Kengne

Copyright (C) 2015 J. N. Seheult et al. This is an open access article distributed under the Creative Commons Attribution License, which permits unrestricted use, distribution, and reproduction in any medium, provided the original work is properly cited.

Background. Poor inpatient glycaemic control has a prevalence exceeding $30 \%$ and results in increased length of stay and higher rates of hospital complications and inpatient mortality. The aim of this study was to improve inpatient glycaemic control by developing an alert system to process point-of-care blood glucose (POC-BG) results. Methods. Microsoft Excel Macros were developed for the processing of daily glucometry data downloaded from the Cobas IT database. Alerts were generated according to ward location for any value less than $4 \mathrm{mmol} / \mathrm{L}$ (hypoglycaemia) or greater than $15 \mathrm{mmol} / \mathrm{L}$ (moderate-severe hyperglycaemia). The Diabetes Team provided a weekday consult service for patients flagged on the daily reports. This system was implemented for a 60 -day period. Results. There was a statistically significant $20 \%$ reduction in the percentage of hyperglycaemic patient-day weighted values $>15 \mathrm{mmol} / \mathrm{L}$ compared to the preimplementation period without a significant change in the percentage of hypoglycaemic values. The time-to-next-reading after a dysglycaemic POC-BG result was reduced by $14 \%$ and the time-to-normalization of a dysglycaemic result was reduced from 10.2 hours to 8.4 hours. Conclusion. The alert system reduced the percentage of hyperglycaemic patient-day weighted glucose values and the time-to-normalization of blood glucose.

\section{Introduction}

Hyperglycaemia and hypoglycaemia are prevalent in the inpatient setting. A recent analysis of hospital glucometry data by Bersoux and Cook from January to December 2012 was conducted on 51.4 million measurements from 2.6 million inpatients. The authors found that the prevalence of hypoglycaemia $(<4 \mathrm{mmol} / \mathrm{L}$ or $70 \mathrm{mg} / \mathrm{dL})$ was $6.1 \%$ in nonICU patients and $5.6 \%$ in ICU patients, while the prevalence of hyperglycaemia (>10 mmol/L or $180 \mathrm{mg} / \mathrm{dL}$ ) was substantially higher at $32 \%$ in non-ICU patients and $28 \%$ in ICU patients. The mean point-of-care blood glucose (POC-BG) was $167 \mathrm{mg} / \mathrm{dL}$ for non-ICU patients and $170 \mathrm{mg} / \mathrm{dL}$ for ICU patients. The authors concluded that increased hospital participation in data collection was needed for the development of optimal practices to manage inpatient dysglycaemia [1].

Numerous studies have shown that improvement in glycemic control results in lower rates of hospital complications in general medicine and surgery patients [2-6]. In one study, newly discovered hyperglycaemia was associated with a higher in-hospital mortality rate (16\%) compared with those patients with a prior history of diabetes (3\%) and subjects with normoglycaemia $(1.7 \%$; both $p<0.01)$ [2].

At the opposite end of the glycaemic scale, studies have shown that in-hospital secondary hypoglycaemia increases inpatient mortality, likelihood of readmission, and length of stay [7-9].

Certain clinical situations increase the risk of dysglycaemia during a hospital admission. These include changes in caloric or carbohydrate intake especially "nil by mouth" status or total parenteral nutrition [10]; use of diabetogenic medications like corticosteroids [11]; failure of medical staff to make adjustments to glycemic therapy based on daily blood glucose (BG) patterns [12]; prolonged use of sliding scale insulin regimens [13]; lack of coordination between insulin therapy, blood glucose monitoring, and meals [14]; issues 
relating to patient transfer from one ward location to another [14]; and medical transcription and dispensing errors [15].

There is evidence that poor inpatient glycaemic control is underrecognized, underreported, and suboptimally managed and that proactive assessment of inpatients' glycaemic status and aggressive treatment approaches are required [1619]. The American Association of Clinical Endocrinologists/American Diabetes Association (AACE/ADA) recommendations stress five key facets: identification of inpatient dysglycaemia; establishing a multidisciplinary team approach to diabetes management in all hospitals; implementation of structured protocols for aggressive control of BG in both ICUs and other hospital settings; educational programs for all hospital personnel caring for people with diabetes; and planning for a smooth transition to outpatient care with appropriate diabetes management $[20,21]$.

Current networked point-of-care technology offers a novel way to address the problem of inpatient dysglycaemia and clinical inertia.

Our hospital conducted an audit of inpatient glucometry results from our Accu-Chek Inform II [22] meters and Cobas IT [23] in October 2012 in order to review the identification and management of patients with hypoglycaemia $(<4 \mathrm{mmol} / \mathrm{L})$ and with moderate $(15-20 \mathrm{mmol} / \mathrm{L})$ and severe (>20 mmol/L) in-hospital hyperglycaemia (see Supplementary Appendix 1 in Supplementary Material available online at http://dx.doi.org/10.1155/2015/807310). 3.4\% of readings were in the hypoglycaemic range and $10 \%$ of readings were in either moderate or severe hyperglycaemic ranges. On average 12 patients per day had POC-BG results in the moderatesevere hyperglycaemic range, with more than 4 times that number having values $>10 \mathrm{mmol} / \mathrm{L}$. The mean time-to-next POC-BG test in a patient with a dysglycaemic value was almost 6 hours with a range of 2 minutes to 33 hours and the mean time-to-normalization of blood glucose after a dysglycaemic result was approximately 10 hours with a range of 1 to 76 hours.

We aimed to develop an alert system where all POC-BG measurements within the moderate-severe hyperglycaemic and hypoglycaemic ranges would be reviewed and notified to the relevant ward teams, nursing staff, and Diabetes Consult service. The metric chosen to analyse glucometry data was the patient-day model, which has been shown to most faithfully reflect the quality of inpatient glycemic control [24]. We aimed to reduce the percentage of patient-day weighted POCBG levels above $15 \mathrm{mmol} / \mathrm{L}$ by at least $20 \%$ with a less than $5 \%$ increase in levels less than $4 \mathrm{mmol} / \mathrm{L}$. We also aimed to reduce the time-to-next-reading and the time-to-normalization after a dysglycaemic result by $20 \%$.

\section{Methods}

2.1. Networked Glucometry System. The glucometers used in this study were the Accu-Chek Inform II system (Roche Diagnostics, Basel, Switzerland) [22]. All hospital glucometers are connected via WLAN to Cobas IT 1000 (Roche Diagnostics, Basel, Switzerland), allowing storage of all information and central management of all meters and data in line with laboratory regulations [23].
2.2. Software Development. Microsoft Excel version 2013 (Microsoft, WA, USA) and Visual Basic version 13 (Microsoft, WA, USA) were used to develop Excel Macros for processing of glucometry results from the Adelaide and Meath Hospital, Dublin, Ireland. An explanation of the Visual Basic Code for the Macros is available in the Supplementary Appendix. A sample anonymised data file and a working version of the Macro-Enabled Workbook is also available online (https:// app.box.com/s/kk0ka29wmuwk855hdbq4yfqvh7nshfnp).

POC-BG data were classified based on local hospital standards: hypoglycaemia (<4 mmol/L), normoglycaemia (4$10 \mathrm{mmol} / \mathrm{L})$, mild hyperglycaemia $(10-15 \mathrm{mmol} / \mathrm{L})$, moderate hyperglycaemia (15-20 mmol/L), and severe hyperglycaemia (>20 mmol/L). Alerts were generated for any hypoglycaemic result and for any result in the moderate-severe hyperglycaemia range.

2.3. Study Design. This study was a 60-day prospective study on the impact of a glucometry alert system on hospital dysglycaemia, started on 11 April 2014. There was no Diabetes Registrar rostered to the project for a 14-day period from 05 May 2014 to 18 May 2014; care proceeded as per the preimplementation phase during this period.

2.3.1. Study Approval. Approval was granted by the hospital Diabetes Consult team, hospital management board, laboratory manager, and information technology department. This project did not require submission to the ethics committee since it was aiming to improve the use of routine clinical data.

2.3.2. Integration with Diabetes Consult Team. Our Diabetes Consult service requested that the cutoff for review of a patient be $15 \mathrm{mmol} / \mathrm{L}$ rather than the ADA/AACE recommendation of $12 \mathrm{mmol} / \mathrm{L}$ since their capacity for reviewing patients was limited to $12-15$ patients per day. There are many cutoffs used for classifying a glucose result as hypoglycaemic. Based on audit data showing that only $3.4 \%$ of our hospital's results were less than $4 \mathrm{mmol} / \mathrm{L}$, the cutoff for hypoglycaemic alerts was set at this level. The touchscreen tablet was used by the Diabetes Consult Registrar to highlight the hospital patients with poor glycaemic control. The Consult Registrar reviewed the patient's history, hospital stay, and insulin regimen. The alert system was used on weekdays only since there was no Consult service on weekends.

2.3.3. Glucose Alerts on Laboratory Information System. The point-of-care manager, Chemical Pathology trainee, or another member of the biochemistry staff performed daily uploads of dysglycaemic results to WinPath (Clinisys Group, Surrey, UK), our laboratory information system. A Glucose Alert (GA) was added to WinPath for every dysglycaemic result with the following comment:

Please review this patient's in-hospital glycaemic control. We have noted episodes of hypoglycaemia $(<4 \mathrm{mmol} / \mathrm{L})$ and/or hyperglycaemia ( $>15 \mathrm{mmol} / \mathrm{L})$ on the point-of-care glucometry alert system. Refer to the hospital guidelines on 
TABLE 1: Summary statistics for pre- and postimplementation periods.

\begin{tabular}{lccc}
\hline Period & Preimplementation & Postimplementation & $p(\alpha=0.05)$ \\
\hline Date range (number of days) & $10-02-2014$ to $10-04-2014(60)$ & $11-04-2014$ to 09-06-2014 (60) & - \\
Mean POC-BG value (mmol/L) & $9.13 \pm 4.57(1.00-33.30)$ & $9.09 \pm 4.47(0.80-33.30)$ & 0.315 \\
Mean patient-day POC-BG value (mmol/L) & $8.22 \pm 3.40(1.80-28.40)$ & $8.09 \pm 3.20(2.80-33.30)$ & 14249 \\
Number of POC-BG readings & 13992 & 5741 & 0.010 \\
Number of patient-days with POC-BG readings & 5984 & 752 & 0.237 \\
Number of patients with POC-BG readings & 816 & 0.186 \\
\hline
\end{tabular}

glycaemic management and/or request a consult from the Hospital Diabetes Team.

The glucose alert uploads to the LIS were performed only on weekdays during the study period.

\subsection{Statistical Methods. Data downloaded from the Cobas IT} 1000 database were analysed using Stata version 13 (Statacorp, TX, USA). POC-BG results were analysed for the pre- and postimplementation periods, from 10 February 2014 to 10 April 2014 and from 11 April 2014 to 9 June 2014, respectively. Results from the acute care wards, emergency department, and pediatrics and outpatient wards were omitted because these have a substantially different patient demographic and/or are not representative of inpatient glycaemic control.

Summary statistics were obtained for the number of POC-BG results every day and the number and percentage of results $<4 \mathrm{mmol} / \mathrm{L}$ and $>15 \mathrm{mmol} / \mathrm{L}$. The percentage of patient-day weighted mean blood glucoses $<4 \mathrm{mmol} / \mathrm{L}$ and $>15 \mathrm{mmol} / \mathrm{L}$ was calculated. Unequal variance one-sided $t$ tests were done to compare mean percentages of hypoglycaemia, hyperglycaemia, hypoglycaemic patient-days, and hyperglycaemic patient-days pre- and postpractice change. The daily mean patient-day weighted POC-BG values were displayed using line graphs with Lowess (locally weighted scatterplot smoothing) lines to show trends over time.

For any POC-BG value $<4 \mathrm{mmol} / \mathrm{L}$ or $>15 \mathrm{mmol} / \mathrm{L}$, the time-to-next-reading and the time-to-normalization of glucose were calculated. If the time between measurements was found to be greater than 72 hours, the patient was assumed to have been either discharged, admitted to ITU, or had serum blood glucoses analysed in the laboratory or on a blood gas analyzer; the time-to-next-reading was taken to be 72 hours in these cases. Comparisons of pre- and postpractice change were made using unequal variance one-sided $t$-tests, histograms, and normal density plots. The proportions of time values greater than 6 hours and 12 hours (time-to-nextreading) or greater than 12 hours and 24 hours (time-tonormalization) were also compared.

Line graphs of the number of POC-BG tests performed per day and the percentage of mean patient-day weighted POC-BG values $>15 \mathrm{mmol} / \mathrm{L}$ were plotted to investigate trends in glycaemic control and glucometry use over weekends.

2.5. Data Security and Confidentiality. The Health Information Technical Standards of the Irish Health Information and Quality Authority (HIQA) were reviewed and followed closely to ensure data security and patient confidentiality [25]. The Head of the Information and Communications Technology Department was also consulted on issues relating to data security.

\section{Results}

In total, 45929 POC-BG results were downloaded from the Cobas IT database for the 120-day analysis period. After removing results from outpatient, paediatric and emergency departments, a total of 13992 results were analyzed for the preimplementation period compared to 14249 in the postimplementation period. There was no statistically significant difference between the numbers of POC-BG values, patientday values, or patients for the two periods (see Table 1).

In total, there were 2023 dysglycaemic values and 272 patients reviewed by the Diabetes Consult Registrar after implementation. This corresponded to approximately 4 new patients and 10 known patients (from consults or from prior review) per day.

The frequency distributions of POC-BG values and mean patient-day POC-BG values by glycaemic level for the preand postimplementation phases are shown in Figure 1. Compared to the preimplementation phase, the percentage of POC-BG values in the moderate-severe hyperglycemia range $(>15 \mathrm{mmol} / \mathrm{L})$ decreased by $6.25 \%(p=0.123)$. There was a $22.6 \%$ reduction in the percentage of patient-day weighted POC-BG values $>15 \mathrm{mmol} / \mathrm{L}(p<0.001)$, as shown in Figure 2. The percentage of hypoglycaemic values and hypoglycaemic patient-day weighted values did not change significantly. While there was a reduction in the percentage of moderate and severe hyperglycaemic values, there was an increase in the percentage of mild hyperglycaemic values but not to the same degree.

A supplementary analysis was performed after omitting the 14-day period from 05 May to 18 May when no Registrar cover was available to review patients flagged by the alert system. After correcting for this protocol deviation, there was a reduction in POC-BG values $>15 \mathrm{mmol} / \mathrm{L}$ from $11.2 \%$ to $10.4 \%(p=0.103)$ and in the percentage of patient-day weighted POC-BG values $>15 \mathrm{mmol} / \mathrm{L}$ from $5.3 \%$ to $4.0 \%$ (31\% relative reduction, $p<0.001$ ) with no change in the percentage of hypoglycaemic values and hypoglycaemic patient-day weighted values ( $p=0.309$ and $p=0.176$ ). Furthermore, there was a greater reduction in the mean hospital patient-day weighted POC-BG from $8.22 \mathrm{mmol} / \mathrm{L}$ to $8.06 \mathrm{mmol} / \mathrm{L}(p<0.01)$. 


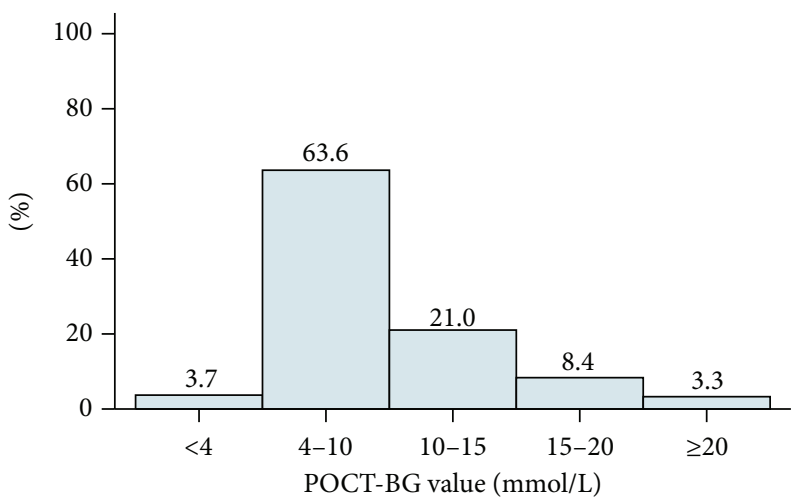

(a)

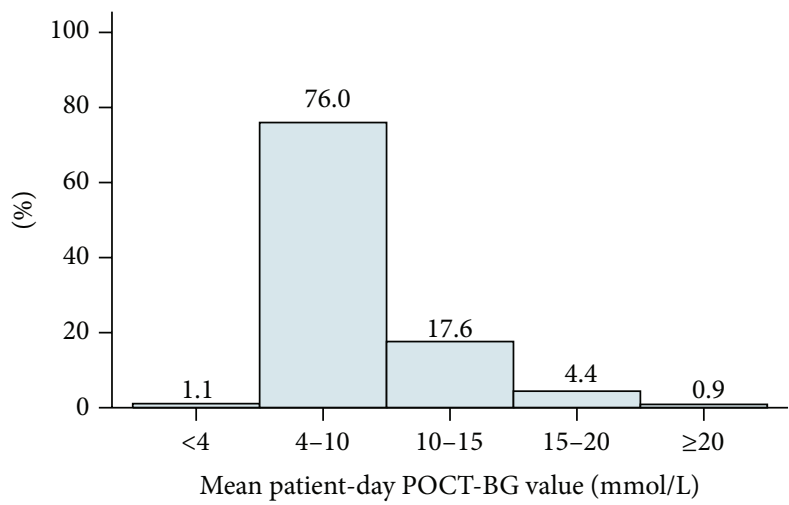

(c)

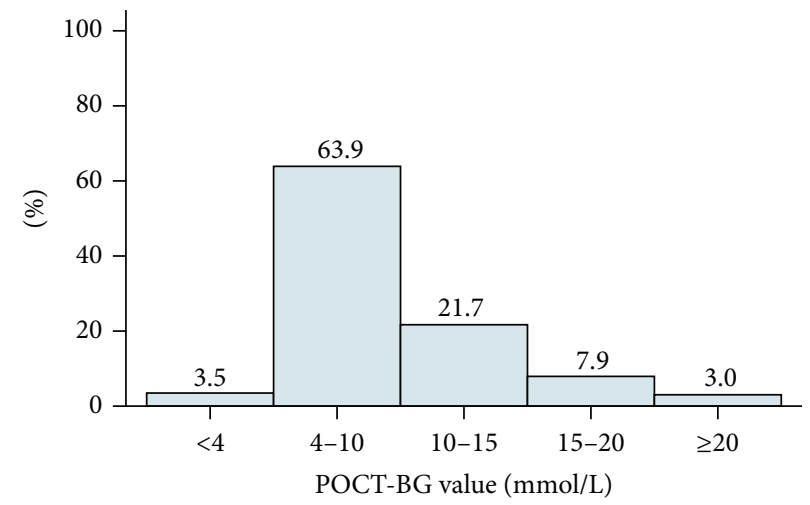

(b)

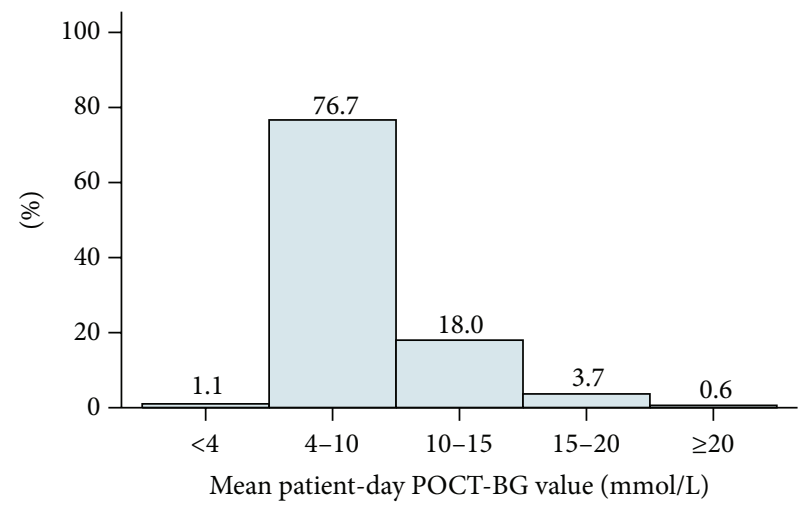

(d)

FIGURE 1: Vertical bar graphs showing the percentage of results by glucose level: (a) preimplementation POC-BG values; (b) postimplementation POC-BG values; (c) preimplementation mean patient-day weighted POC-BG values; and (d) postimplementation mean patient-day weighted POC-BG values.

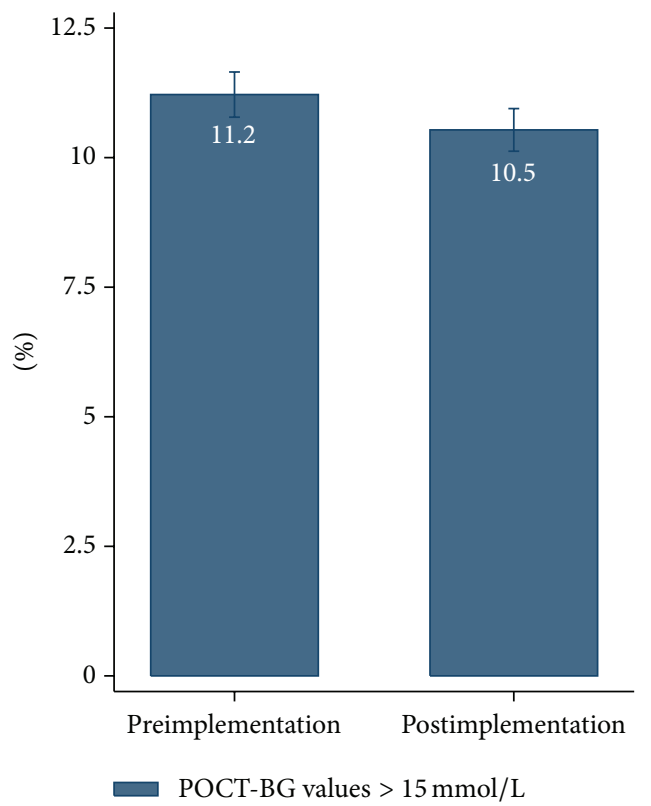

(a)

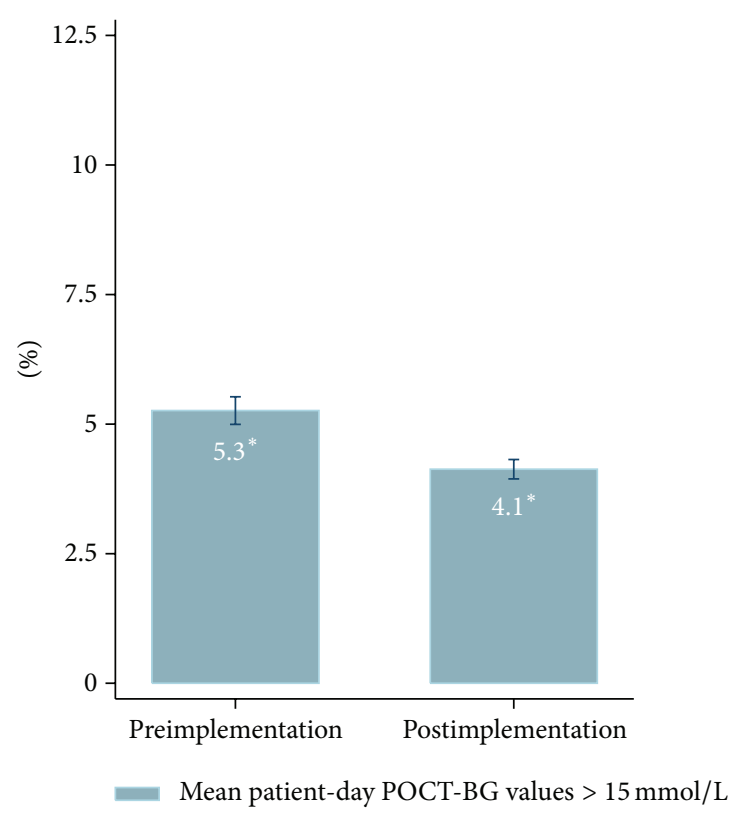

(b)

FIGURE 2: Vertical bar graphs showing the percentage of hyperglycaemic ( $>15 \mathrm{mmol} / \mathrm{L})$ (a) POC-BG values and (b) mean patient-day weighted POC-BG values. There was a significant reduction (greater than 20\%) in mean patient-day weighted POC-BG values $>15 \mathrm{mmol} / \mathrm{L}$ after implementation of the alert system. ${ }^{*} p<0.05$. 


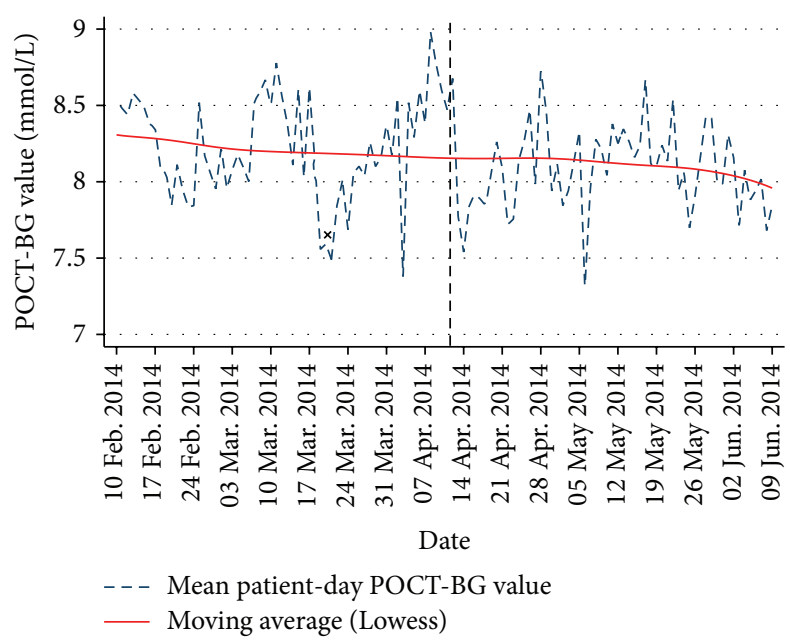

FIGURE 3: Line graph and Lowess moving average of hospital mean patient-day weighted POC-BG value over time. There was a significant decrease after the date of implementation of the alert system, as highlighted by the dashed line. " $\mathrm{x}$ " marks the time period when new hospital guidelines were implemented for the management of inpatient dysglycaemia.

Figure 3 shows the daily change in mean patient-day weighted POC-BG values for the entire study. During the preimplementation phase, the mean hospital value was approximately $8.22 \mathrm{mmol} / \mathrm{L}$. There was a statistically significant reduction to $8.09 \mathrm{mmol} / \mathrm{L}$ after the alert system was implemented $(p<0.01)$, as shown in Table 1 . The mean patient-day weighted POC-BG value for the hospital plateaued between 05 May and 19 May when the Registrar was not available to review patients. There was a similar trend in mean POC-BG value but this was not statistically significant (not shown here). The " $\mathrm{x}$ " in Figure 3 marks the time period when new guidelines were implemented for the management of inpatient dysglycaemia. This coincided with a 1-2-week reduction in the mean patient-day weighted POC$B G$ value for the hospital; however, the hospital mean reached its previous baseline after this short-lived change.

There was a significant $14 \%$ reduction in the mean time-to-next-reading, from 5.1 hours to 4.4 hours $(p<$ $0.01)$. The density histogram shows a reduction in values over 6 hours, with a shift of the probability density plot to the left (see Figure 4). $25.2 \%$ of all times were greater than 6 hours prior to implementation compared to $20.8 \%$ after implementation $(p<0.001)$. The mean time-tonormalization of a dysglycaemic POC-BG result decreased by $19 \%$ from 10.2 hours to 8.3 hours $(p<0.01)$. The density histogram shows a fall in the number of values greater than 12 hours for this parameter compared to preimplementation. As shown in Table 2, prior to implementation, $25.0 \%$ of time-to-normalization values were greater than 12 hours with $7.4 \%$ greater than 24 hours compared to $21.5 \%$ and $5.1 \%$, respectively, after implementation $(p<0.05)$.

The average number of POC-BG tests performed per day was approximately 230 . There was a clear trend towards a lower number of tests on weekends with $240 \pm 32$ tests
TABLE 2: Proportion of values for time-to-next-reading and time-tonormalization values above specified thresholds.

\begin{tabular}{|c|c|c|c|}
\hline \multicolumn{4}{|c|}{ Time-to-next-reading after dysglycaemic result } \\
\hline & Preimplementation & Postimplementation & $p$ (1-tailed) \\
\hline$>6$ hours & $25.20 \%$ & $20.80 \%$ & 0.0007 \\
\hline$>12$ hours & $8.60 \%$ & $6.00 \%$ & 0.0004 \\
\hline \multicolumn{4}{|c|}{ Time-to-normalization of dysglycaemic result } \\
\hline & Preimplementation & Postimplementation & $p$ \\
\hline$>12$ hours & $25.00 \%$ & $21.50 \%$ & 0.032 \\
\hline$>24$ hours & $7.40 \%$ & $5.10 \%$ & 0.016 \\
\hline
\end{tabular}

performed on weekdays compared to $223 \pm 33$ tests performed on weekends ( $p=0.008$ ), highlighted by the red bars on Figure 5. Towards the end of the implementation period, there was a rise in the total number of POC-BG tests performed above the previous hospital average.

A trend towards increased proportions of dysglycaemic results on weekends was also noted (indicated by the red bars on Figure 6); this trend was exaggerated in the postimplementation phase. However, there was no statistically significant difference in the proportion of hyperglycaemic results on weekends compared to weekdays. There was also a significant reduction in the coefficient of variation of the percentage of hyperglycaemic results from $40 \%$ to $35 \%$; this is visually evident by the stabilization of postimplementation values.

\section{Discussion}

A comprehensive alert system was devised using Visual Basic code and Microsoft Excel Macros to process data and provide charts and tables representing daily glycaemic control for inpatient wards in the hospital. This data was used to provide alerts to hospital teams via the Laboratory Information System and to alert the Diabetes Consult service about patients requiring urgent review.

Data from the preimplementation period agreed with results from our audit in 2012, showing that the percentage of hypoglycaemic results was between 3 and $4 \%$, the percentage of hyperglycaemic results in the mild range was $\sim 20 \%$, and the percentage of results in the moderate-severe hyperglycaemic was approximately $12 \%$. This shows that our rates of dysglycaemia were relatively stable over the previous year.

The implementation of the glucometric alert system achieved our target of a $20 \%$ reduction in the percentage of patient-day weighted POC-BG values above $15 \mathrm{mmol} / \mathrm{L}$, the main metric used in this study. Importantly, the percentage of hypoglycaemic results did not change significantly. One of the known complications of strict glycaemic control is an increase in secondary hypoglycaemic events. While addressing the problem of hyperglycaemia, a related goal was to ensure that the proportion of hypoglycaemic results either reduced or remained unchanged and this was achieved by the alert system.

Hypoglycaemic events tend to be managed more proactively in hospital compared to hyperglycaemia for a couple of reasons. Firstly, patients are usually symptomatic 


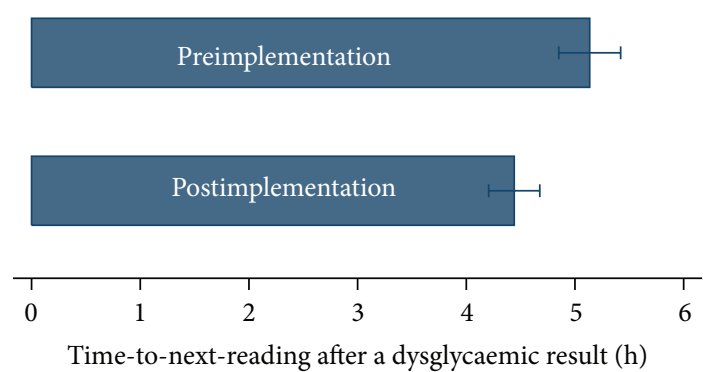

(a)

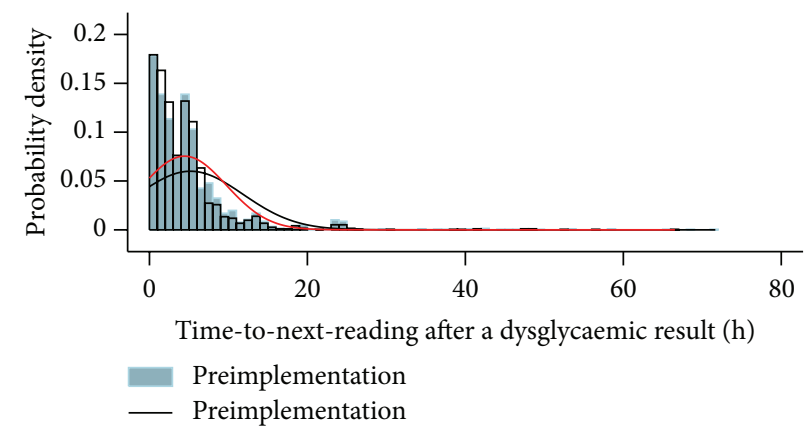

(c)
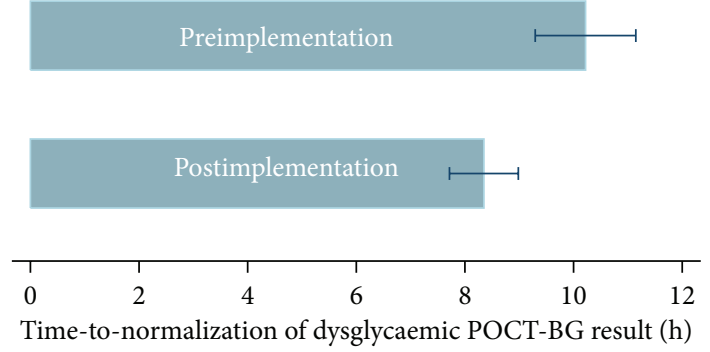

(b)

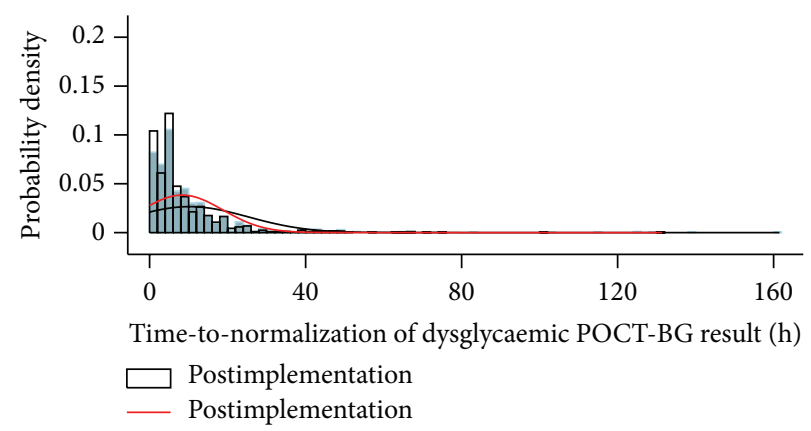

(d)

FiguRE 4: Bar graphs showing mean and 95\% CI of (a) time-to-next-reading after dysglycaemic results and (b) time-to-normalization of a dysglycaemic result, before and after implementation. Histograms with probability density plots of (c) time-to-next-reading after a dysglycaemic result and (d) time-to-normalization of a dysglycaemic result, before and after implementation.

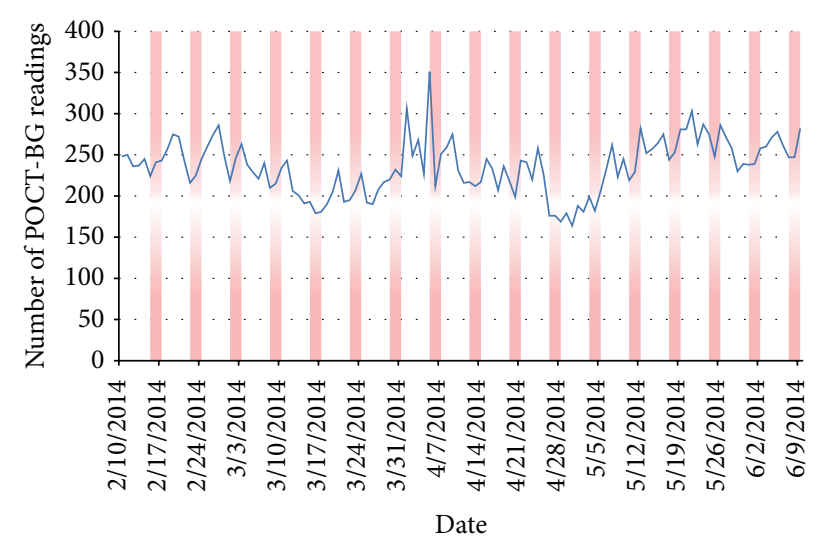

FIGURE 5: Line graph showing the number of POC-BG tests done by date in all ward locations analysed. Red area bars represent weekends.

when blood glucose levels fall below $3 \mathrm{mmol} / \mathrm{L}$ unless they have had frequent hypoglycaemic events in the past or are on beta-blocker therapy, rendering them "hypoglycaemia unaware." Secondly, the management of hypoglycaemia is much simpler in the initial stages with oral or intravenous glucose formulations or intramuscular glucagon, with an almost immediate rise in blood glucose levels. Hospital and nursing staff have a tendency to be more permissive of mild-moderate hyperglycaemia, employing a watch and wait approach allowing time for the patient's own insulin or hypoglycaemic regimen to work and waiting for consecutive

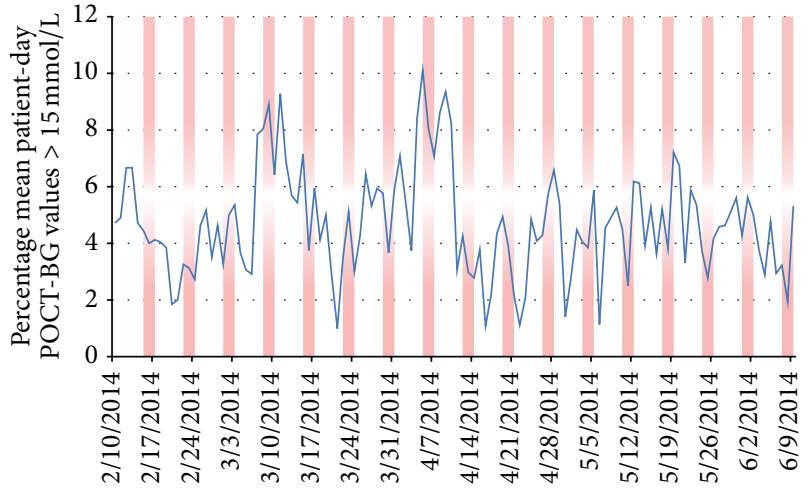

Date

FIGURE 6: Line graph showing the percentage of mean patient-day weighted POC-BG results greater than $15 \mathrm{mmol} / \mathrm{L}$ by date in all ward locations analysed. Red area bars represent weekends.

results to be above some arbitrary threshold before alerting hospital teams. This is evident by the much higher rates of hyperglycaemia compared to hypoglycaemia.

The most important benefit of the alert system seems to be the improvement in the time-to-normalization of a dysglycaemic result. This coincided with more frequent POC-BG testing if a result was dysglycaemic, manifested by an increased number of POC-BG tests performed after implementation and a reduction in the time-to-next-reading. The $19 \%$ reduction in time-to-normalization and the $14 \%$ reduction in mean time-to-next-reading fell short of our 
$20 \%$ target; however, there was a $31 \%$ relative reduction in time-to-normalization values greater than 24 hours and a $30 \%$ relative reduction in time-to-next-reading values greater than 12 hours. This means that the alert system successfully reduced significant outliers seen in the preimplementation phase. Likely explanations are that the Diabetes Consult service reviewed patients in a more timely fashion and also that more physiological insulin regimens were utilized to normalize dysglycaemic results.

During our 2012 audit, we noticed that the weekend glycaemic control was worse than that seen on weekdays, with higher levels of moderate-severe hyperglycaemia. This problem was inadequately addressed in this study because of a lack of staff coverage on the Diabetes Consult service on weekends. Our results show a trend towards lower numbers of POC-BG tests on weekends; the number of tests performed increased towards the end of the implementation period for unknown reasons. There were also frequent spikes in the percentage of patient-day weighted hyperglycaemic results on weekends and this trend was exaggerated during the implementation phase, likely due to the fact that weekday glycaemic control was improved significantly. The trends observed were not statistically significant; however, they do mirror the trends seen during our previous audit and warrant further investigation and action plans.

Figure 6 shows that the variability in the percentage of hyperglycaemic values was reduced. Before implementation, two peaks in hyperglycaemia were noted; these peaks may be due to the patient population in the hospital at the time. On 21 March 2014, new guidelines for management of dysglycaemia were launched in the hospital and a presentation was given to general medical and surgical teams outlining these guidelines. It is likely that glycaemic control improved in the week following the launch of these new guidelines and that the baseline rate of dysglycaemia in the preimplementation period would have been higher than what was witnessed.

The major limitations of this study related to personnel, financial, and technical issues. The Diabetes Consult service was run by a team of one Consultant and one Registrar and the number of patients that could be reviewed on a daily basis was limited to 10-15. Also, a Consult service based on flagged dysglycaemic results was not provided on weekends and public holidays. It is likely that the percentage of dysglycaemic patient-days would have been significantly lower with more staffing cover. Funding for specialized software development was not available and all technical expertise was local and limited in scope. Uploads to the Laboratory Information System and to the tablets were done twice daily, meaning that there was a significant lag between the time when the glucometry test was performed and the time when the result was uploaded or reviewed by the Diabetes Consult Registrar. Further integration with the current IT framework would allow for instantaneous uploads to the LIS and updates to the interactive reports and charts on the tablets in a push notification fashion.

While this study showed an improvement in glycaemic control, no clinical correlations could be made. Determining changes in length of stay, inpatient mortality, and cost of care by using the alert system was outside the scope of the study and would require significant input by hospital administration. The number of patients in hospital with either a primary or secondary diagnosis of diabetes would be a key metric to monitor over time and control for. Estimates of dysglycaemia could then be corrected for the number of diabetics in each ward location. Additionally, the alert system was only implemented for a 60-day period in this pilot study. Due to fluctuations in the patient profile admitted to hospital, it is possible that the improvements in glycaemic control are artefactual.

Potential areas for improvement would be in the user interface and alert software as well as in the current networking infrastructure. Direct access to the Cobas IT webserver would allow more frequent automatic downloads of data to a network shared folder, which can be accessed on demand by the tablets or any computer in the hospital. Measurement of ketones using point-of-care devices has become more important since the Joint British Diabetes Society (JBDS) issued guidelines in March 2010 recommending ketone (betahydroxybutyrate) measurement in the management of DKA [26]. Ketone results from point-of-care devices should also form part of a comprehensive alert system geared especially towards type 1 diabetics.

To determine whether there is a reduction in hospital cost, length of stay, and improved outcomes associated with improved glycaemic control due to the alert system, a sixmonth to one-year study is needed, with comparison to the previous year. Key metrics like length of stay and inpatient mortality, as well as perioperative outcomes for patients with a primary or secondary diagnosis of diabetes, will need to be recorded rigorously. The longer timeframe of the study will negate the effect of artefactual fluctuations in glycaemic control that are caused by the number of diabetics in hospital and other factors.

\section{Summary and Conclusions}

An automated alert system that allowed the use of sitespecific level and time-based criteria for the identification of dysglycaemic results and generation of alerts was successfully developed and deployed in our medium-large sized tertiary care facility. There was a significant improvement in our hospital's glycaemic control, with a reduction in the proportion of hyperglycaemic patient-days and no rebound increase in secondary hypoglycaemia. The alert system also improved the frequency with which glucometry tests were performed in patients with recognized out-of-control glucose results and improved the time-to-normalization of dysglycaemic results. The percentage of patient-day weighted results $>15 \mathrm{mmol} / \mathrm{L}$ was reduced by greater than $20 \%$ and while the reduction in the time-to-normalization of dysglycaemic POC-BG results fell short of our $20 \%$ target, we successfully reduced the proportion of values greater than 12 hours by more than $20 \%$. Certain trends in glucometry testing and glycaemic control were also observed, with lower numbers of tests performed and spikes in hyperglycaemia on weekends. Longer-term studies will enable us to determine whether improvements in glycaemic control correlate with improvements in in-hospital 
morbidity and mortality and reductions in length of stay and hospital costs.

\section{Conflict of Interests}

The authors declare that there is no conflict of interests regarding the publication of this paper.

\section{Acknowledgments}

The authors would like to thank the Adelaide and Meath Hospital and Trinity College Dublin for providing financial support for this project.

\section{References}

[1] S. Bersoux, C. B. Cook, G. L. Kongable, and J. Shu, "Trends in glycemic control over a 2-year period in 126 US hospitals," Journal of Hospital Medicine, vol. 8, no. 3, pp. 121-125, 2013.

[2] G. E. Umpierrez, S. D. Isaacs, N. Bazargan, X. You, L. M. Thaler, and A. E. Kitabchi, "Hyperglycemia: an independent marker of in-hospital mortality in patients with undiagnosed diabetes," Journal of Clinical Endocrinology and Metabolism, vol. 87, no. 3, pp. 978-982, 2002.

[3] M. Y. Rady, D. J. Johnson, B. M. Patel, J. S. Larson, and R. A. Helmers, "Influence of individual characteristics on outcome of glycemic control in intensive care unit patients with or without diabetes mellitus," Mayo Clinic Proceedings, vol. 80, no. 12, pp. 1558-1567, 2005.

[4] P.-C. Chen, S.-K. Chua, H.-F. Hung et al., "Admission hyperglycemia predicts poorer short- and long-term outcomes after primary percutaneous coronary intervention for ST-elevation myocardial infarction," Journal of Diabetes Investigation, vol. 5, no. 1, pp. 80-86, 2014.

[5] F. J. Pasquel, D. Smiley, R. Spiegelman, E. Lin, L. Peng, and G. E. Umpierrez, "Hyperglycemia is associated with increased hospital complications and mortality during parenteral nutrition," Hospital Practice, vol. 39, no. 2, pp. 81-88, 2011.

[6] F. J. Pasquel, R. Spiegelman, M. Mccauley et al., "Hyperglycemia during total parenteral nutrition: an important marker of poor outcome and mortality in hospitalized patients," Diabetes Care, vol. 33, no. 4, pp. 739-741, 2010.

[7] A. Zapatero, R. Gómez-Huelgas, N. González et al., "Frequency of hypoglycemia and its impact on length of stay, mortality, and short-term readmission in patients with diabetes hospitalized in internal medicine wards," Endocrine Practice, vol. 20, no. 9, pp. 870-875, 2014.

[8] K. G. Brodovicz, V. Mehta, Q. Zhang et al., "Association between hypoglycemia and inpatient mortality and length of hospital stay in hospitalized, insulin-treated patients," Current Medical Research \& Opinion, vol. 29, no. 2, pp. 101-107, 2013.

[9] K. Nirantharakumar, T. Marshall, A. Kennedy, P. Narendran, K. Hemming, and J. J. Coleman, "Hypoglycaemia is associated with increased length of stay and mortality in people with diabetes who are hospitalized," Diabetic Medicine, vol. 29, no. 12, pp. e445-e448, 2012.

[10] M. Curll, M. DiNardo, M. Noschese, and M. T. Korytkowski, "Menu selection, glycaemic control and satisfaction with standard and patient-controlled consistent carbohydrate meal plans in hospitalised patients with diabetes," Quality and Safety in Health Care, vol. 19, no. 4, pp. 355-359, 2010.
[11] A. C. Donihi, D. Raval, M. Saul, M. T. Korytkowski, and M. A. DeVita, "Prevalence and predictors of corticosteroid-related hyperglycemia in hospitalized patients," Endocrine Practice, vol. 12, no. 4, pp. 358-362, 2006.

[12] M. Noschese, A. C. Donihi, G. Koerbel et al., "Effect of a diabetes order set on glycaemic management and control in the hospital," Quality and Safety in Health Care, vol. 17, no. 6, pp. 464-468, 2008.

[13] G. E. Umpierrez, A. Palacio, and D. Smiley, "Sliding scale insulin use: myth or insanity?" American Journal of Medicine, vol. 120, no. 7, pp. 563-567, 2007.

[14] R. Hellman, "Patient safety and inpatient glycemic control: translating concepts into action," Endocrine Practice, vol. 12, no. 3, pp. 49-55, 2006.

[15] R. Hellman, "A systems approach to reducing errors in insulin therapy in the inpatient setting," Endocrine Practice, vol. 10, supplement 2, pp. 100-108, 2004.

[16] M. T. Korytkowski, "In-patient management of diabetes: controversies and guidelines," Indian Journal of Endocrinology and Metabolism, vol. 17, supplement 3, pp. S630-S635, 2013.

[17] K. Hawkins, A. C. Donihi, and M. T. Korytkowski, "Glycemic management in medical and surgical patients in the Non-ICU setting," Current Diabetes Reports, vol. 13, no. 1, pp. 96-106, 2013.

[18] M. E. Desimone, G. E. Blank, M. Virji et al., "Effect of an educational inpatient diabetes management program on medical resident knowledge and measures of glycemic control: a randomized controlled trial," Endocrine Practice, vol. 18, no. 2, pp. 238-249, 2012.

[19] M. Korytkowski, M. Dinardo, A. C. Donihi, L. Bigi, and M. de Vita, "Evolution of a diabetes inpatient safety committee," Endocrine Practice, vol. 12, no. 3, pp. 91-99, 2006.

[20] E. S. Moghissi, M. T. Korytkowski, M. DiNardo et al., "American Association of Clinical Endocrinologists and American Diabetes Association consensus statement on inpatient glycemic control," Endocrine Practice, vol. 15, no. 4, pp. 353-369, 2009.

[21] E. S. Moghissi, M. T. Korytkowski, M. DiNardo et al., "American Association of Clinical Endocrinologists and American Diabetes Association consensus statement on inpatient glycemic control," Diabetes Care, vol. 32, no. 6, pp. 1119-1131, 2009.

[22] Roche Diagnostics, AccuChek Inform II System, 2014, http:// www.Accu-Chekinformii.com/.

[23] Roche Diagnostics, “Cobas IT solutions," June 2014, http://www .roche-diagnostics.co.in/Products/Pages/COBASITsolutions .aspx.

[24] P. A. Goldberg, J. E. Bozzo, P. G. Thomas et al., "'Glucometrics'assessing the quality of inpatient glucose management," Diabetes Technology \& Therapeutics, vol. 8, no. 5, pp. 560-569, 2006.

[25] Health Information and Quality Authority, International Review of Information Security in Health and Social Care, 2012, http://www.hiqa.ie/publications/international-review-information-security-health-and-social-care.

[26] M. W. Savage, K. K. Dhatariya, A. Kilvert et al., "Joint British Diabetes Societies guideline for the management of diabetic ketoacidosis," Diabetic Medicine, vol. 28, no. 5, pp. 508-515, 2011. 


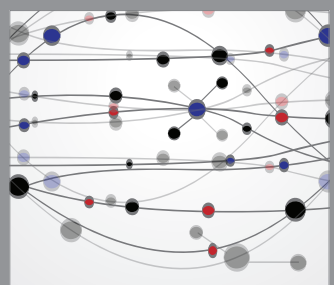

The Scientific World Journal
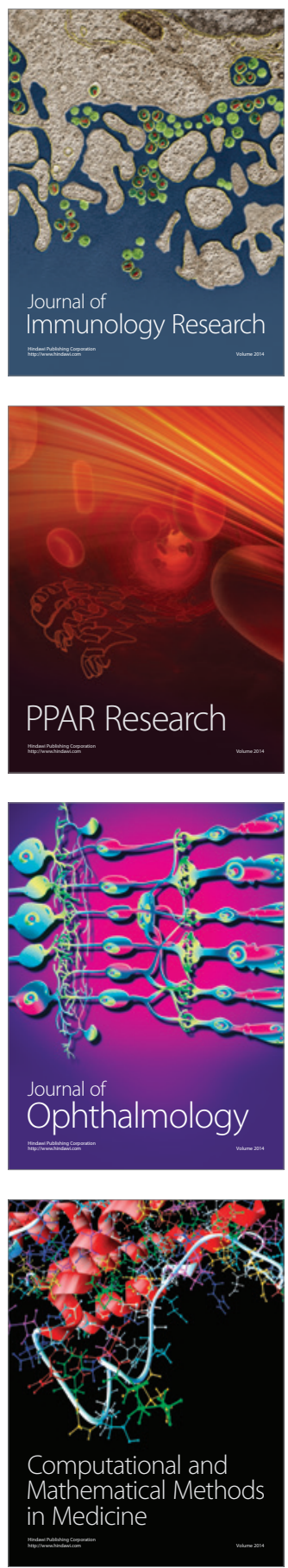

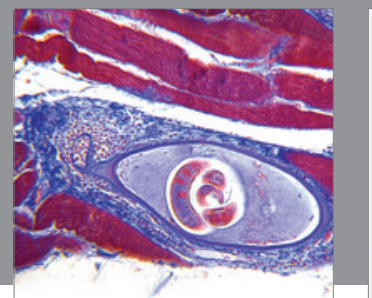

Gastroenterology

Research and Practice
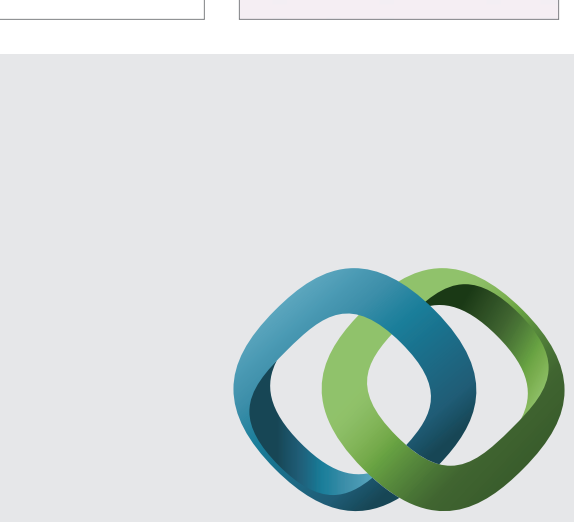

\section{Hindawi}

Submit your manuscripts at

http://www.hindawi.com
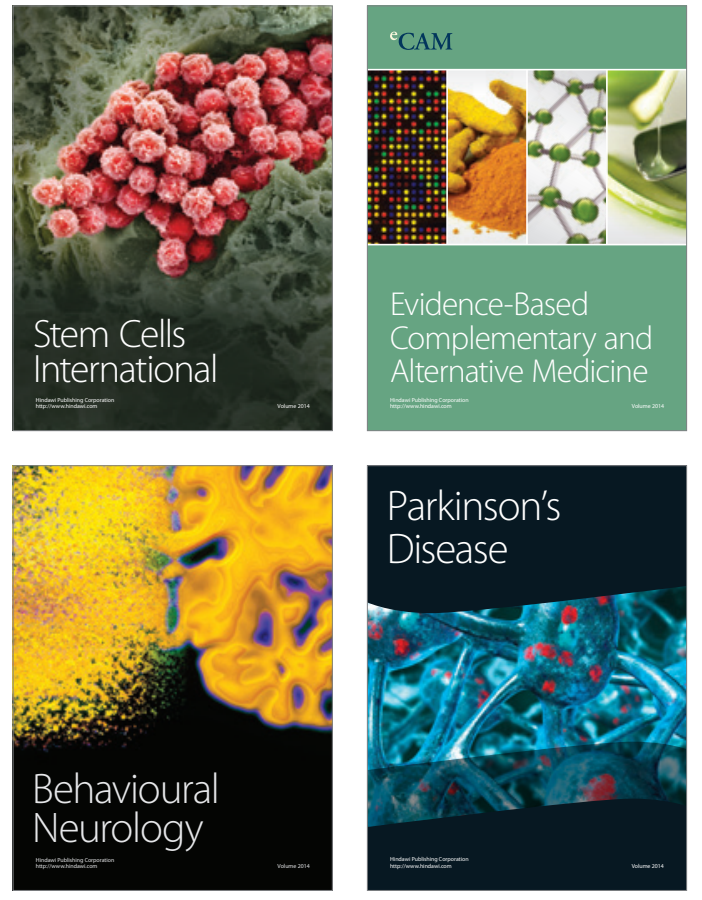
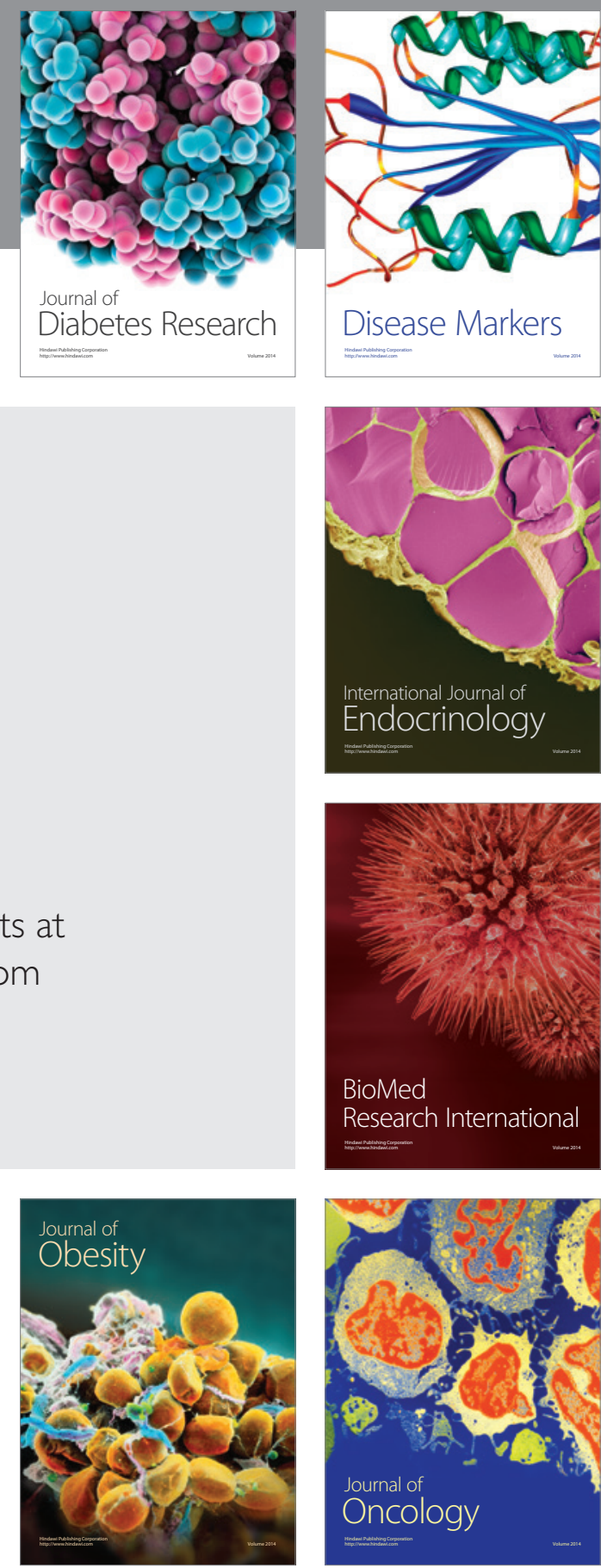

Disease Markers
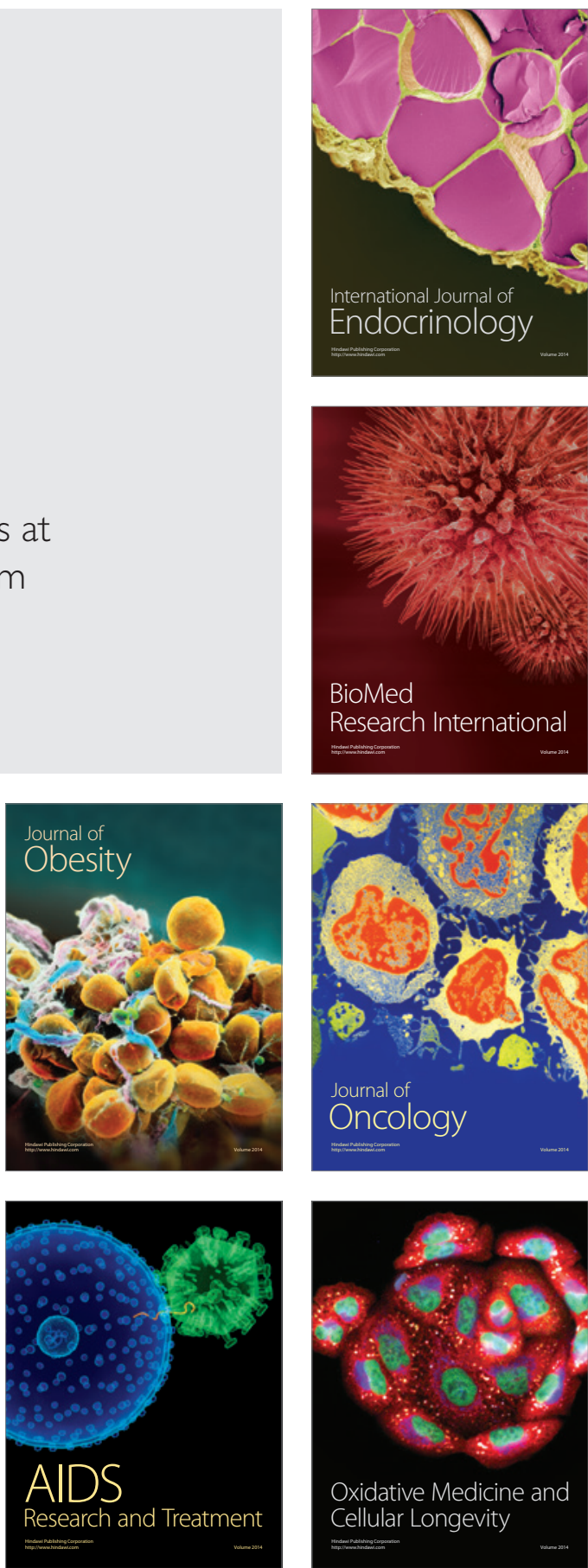